\title{
Recurrent Abdominal Pain Caused by Sorbitol Malabsorption
}

\author{
Cátia Granja* \\ Department of Pediatrics, Hospital Distrital da Figueira da Foz, Portugal
}

*Corresponding author: Cátia Granja, Department of Pediatrics, Hospital

Distrital da Figueira da Foz, Portugal.

Received Date: February 11, 2021

Published Date: March 16, 2021

\begin{abstract}
Sorbitol is a carbohydrate, a monosaccharide polyalcohol, naturally present in fruits and juices. It is also widely used as a "sugar-free" substitute sweetener in the food industry, especially in sweets, chewing gum and diet products $[1,2]$.

The incidence of sorbitol intolerance in the general population is estimated at 8 to $12 \%$ and are frequently combined with adverse food reactions, food allergies, and irritable bowel syndrome (IBS). Sorbitol undergoes only slight intestinal resorption by passive diffusion and absorption is dose and concentration related. Sorbitol can be transformed into fructose within the intestine, blocking GLUT-5 transporter, so the symptoms are the same as those of fructose malabsorption [2,3]. This results in the typical cardinal symptoms of postprandial flatulence, nausea, meteorism, diarrhea, and nonspecific abdominal pain [4].

Sorbitol $\mathrm{H} 2$ breath test is effective in detecting small bowel damage with a relevant reduction of absorption surface. In carbohydrate malabsorption false positive tests for small intestinal bacterial overgrowth may occur due to colonic fermentation and production of gas. In gastrointestinal motor disorders, delayed gastric emptying may cause false negative tests, and rapid transit through small bowel may result in false positive breath tests, false positive results may also occur if the subject does not adhere to a low fiber diet the day before the test [5].

Keywords: Sorbitol malabsorption; Chronic diarrhea; Abdominal pain; Sugar-free products
\end{abstract}

\section{Case Presentation}

A 12 years-old girl, with a history of chronic abdominal pain, periumbilical cramping occurring several times each week, diarrhea lasting 2-3 days, with several years of evolution, interpreted as IBS. About two months ago, the condition worsened with spasmodic pain in the lower quadrants and multiple diarrheal dejections, including nocturnal ones. There was no history of weight loss, fevers, rectal bleeding, fatigue, or increased flatulence. Growth and development had been normal. Family history was noncontributory. Results of the physical examination were within normal limits. Abdominal, renal and pelvic ultrasound without changes. Parasitological study of feces, fecal calprotectin, blood count, blood glucose, inflammatory parameters and thyroid function was normal, and screening of celiac disease was negative. Further history subsequently obtained revealed that for the previous years the patient had been chewing one to two pieces of sugar-free sorbitol-containing gum several times each week and high consumption of light drinks and diet cereal bars that she used to improve motion sickness. A therapeutic trial eliminating sugar-free products for six weeks resulted in the disappearance of the abdominal cramping.

\section{Discussion}

It has long been known that many short-chain carbohydrates can induce abdominal symptoms that are similar to those in patients with IBS $[4,6]$. Up to $80 \%$ of IBS patients believe that their symptoms are diet-related, of which three quarters are possibly related to intolerance to carbohydrates [7]. The diagnosis depends 
on the exclusion of organic diseases and therapeutic strategies are drawn up depending on the dominant symptom and clinical history.

The authors intend to warn of the importance of food history and suggest the evaluation of patients with gastrointestinal complaints should include a careful investigation of the use of products containing sorbitol.

\section{Acknowledgement}

None.

\section{Conflict of Interest}

No conflict of interest.

\section{References}

1. Berni Canani R, Pezzella V, Amoroso A, Cozzolino T, Di Scala C, et al. (2016) Diagnosing and Treating Intolerance to Carbohydrates in Children. Nutrients 8(3): 157.
2. Raithel M, Weidenhiller M, Hagel AF-K, Hetterich U, Neurath MF, et al. (2013) The malabsorption of commonly occurring mono and disaccharides: Levels of investigation and differential diagnoses. Dtsch Arztebl 110(46): 775-782.

3. Montalto M, Gallo A, Ojetti V, Gasbarrini A (2013) Fructose, trehalose and sorbitol malabsorption. Eur Rev Med Pharmacol Sci 17(Suppl 2): 26-29.

4. Plunkett A, Beattie RM (2005) Recurrent abdominal pain in childhood. J R Soc Med 98(3): 101-106.

5. Rana SV, Malik A (2014) Breath tests and irritable bowel syndrome. World J Gastroenterol 20(24): 7587-7601.

6. Gibson PR (2017) History of the low FODMAP diet. J Gastroenterol Hepatol 32(Suppl 1): 5-7.

7. Mansueto P, Seidita A, D’Alcamo A, Carroccio A (2015) Role of FODMAPs in Patients With Irritable Bowel Syndrome. Nutr Clin Pract 30(5): 665682. 